\title{
PROPUESTA DE DECLARACIÓN DEL ESPECTRO ELECTROMAGNÉTICO PARA ECUADOR
}

\author{
PROPOSAL OF ELECTROMAGNETIC SPECTRUM DECLARATION FOR ECUADOR
}

\author{
Myrian Herrera $^{1}$; Luis Porras ${ }^{1}$; Carlos Estrella ${ }^{123}$. \\ ${ }^{1}$ INSTITUTO GEOGRÁFICO MILITAR, QUITO, ECUADOR. Seniergues E4-676 y Gral. Telmo Paz \\ y Miño, Quito, Ecuador. E-mail: myrian.herrera@mail.igm.gob.ec; luis.porras@mail.igm.gob.ec; carlos. \\ estrella@mail.igm.gob.ec \\ ${ }^{2}$ GRUPO DE INVESTIGACIÓN GEOESPACIAL, UNIVERSIDAD DE LAS FUERZAS ARMADAS, \\ "ESPE", SANGOLQUÍ, ECUADOR. Av. General Rumiñahui S/N, Sangolquí, Ecuador. E-mail: \\ carlosmestrellap@gmail.com \\ ${ }^{3}$ INSTITUTO GULICH, UNIVERSIDAD NACIONAL DE CÓRDOBA/COMISIÓN NACIONAL DE \\ ACTIVIDADES ESPACIALES, CÓRDOBA, ARGENTINA. Ruta Provincial C45 a 8 Km CP 5187 Falda \\ del Cañete, Córdoba, Argentina. E-mail: carlosmestrellap@gmail.com
}

\section{RESUMEN}

El presente trabajo muestra el estado del arte respecto de los diferentes límites asignados a cada rango del espectro electromagnético por distintos autores y organismos internacionales, y busca a través de esta revisión establecer una única "Propuesta de declaración del espectro electromagnético para Ecuador", que claramente identifique estos límites y aporte a la universalización de criterios y lenguaje, comparación de resultados interinstitucionales e internacionales de forma clara y precisa, y sirva como aporte para identificar de manera objetiva las secciones no atendidas del espectro, para la adecuada planificación, uso y, gestión de este recurso, por parte de los diferentes actores e instituciones públicas y principalmente en atención a los ejes de Seguridad y Defensa Nacional.

Palabras claves: Espectro electromagnético, gestión del espectro electromagnético, propuesta de declaración espectro electromagnético.

\begin{abstract}
The present work shows the state of art about the different limits assigned to each range of the electromagnetic spectrum by different authors and international organizations, and searches through the bibliographic review to establish a single "Proposed declaration of the electromagnetic spectrum for Ecuador", that clearly identify these limits and contribute to the universalization of criteria and language, comparison of interinstitutional and international results in a clear and precise way, and serve as a contribution to identify in an objective way the sections not attended of the spectrum, for the proper planning, use and management of this resource, by the different actor and public institutions and mainly in attention to the axes of Security and National Defense.
\end{abstract}

Keywords: Electromagnetic spectrum, management of the electromagnetic spectrum, electromagnetic spectrum statement proposal. 


\section{INTRODUCCIÓN}

El espectro electromagnético es el rango completo de todas las radiaciones electromagnéticas (National Aeronautics and Space Administration, 2011), Radio, Microondas, Infrarrojo, Visible, Ultravioleta, Rayos X y Rayos Gamma (National Aeronautics and Space Administration, 2014), y es considerado como un recurso económico y técnico, por lo que su eficaz utilización puede tener un impacto elevado en la prosperidad de un país (Unión Internacional de Telecomunicaciones, 2009).

$\mathrm{Su}$ adecuada utilización respecto de la distribución energética de las ondas electromagnéticas, el entendimiento claro de su comportamiento e interacción con los objetos, y la implementación de buenas prácticas de gestión y reglamentación respecto de uso, posibilita que los usuarios puedan mejorar sus aplicaciones, servicios, tecnologías y métodos de medición entre otros posibles beneficios.

En tal razón y con el objetivo de aportar a los procesos de buenas prácticas de gestión y reglamentación en el uso de este recurso, se plantea una "Propuesta de declaración del espectro electromagnético para Ecuador", que contribuya con la delimitación y estandarización de los rangos de las sub clasificaciones del espectro, sirviendo como guía de información para las instituciones y usuarios relacionados con la planificación, uso y gestión de este recurso, en aporte tanto a la universalización de criterios y lenguaje, así como punto de partida para la comparación de resultados a nivel interinstitucionales e internacionales fruto del uso de este recurso y entre otros beneficios el ayudar a enfocar de manera objetiva los esfuerzos de gestión en aquellas secciones no atendidas del espectro.

\section{DEFINICIÓN DEL ESPECTRO ELECTROMAGNÉTICO}

Las ondas electromagnéticas son la propagación simultánea de los campos eléctricos y magnéticos producidos por una carga eléctrica en movimiento, campos que son mutuamente perpendiculares entre sí y perpendiculares a la dirección de propagación de las ondas, y que a su vez, conforman la radiación electromagnética (Fontal, Suárez, Reyes, Bellandi, Contreras, \& Romero, 2005) (Ver Figura 1).

Las ondas electromagnéticas transportan energía electromagnética y movimiento desde alguna fuente a un receptor y se desplazan en el vacío a la velocidad de la luz c $=299.792$ $\mathrm{km} / \mathrm{s}$, los parámetros que caracterizan este movimiento son la longitud de onda $(\lambda)$ y la frecuencia $(v)$ de las ondas electromagnéticas, que se relacionan mediante la expresión $\mathrm{c}=\lambda v$, que permite determinar su energía, su visibilidad, su poder de penetración y otras características (Fontal, Suárez, Reyes, Bellandi, Contreras, \& Romero, 2005). 


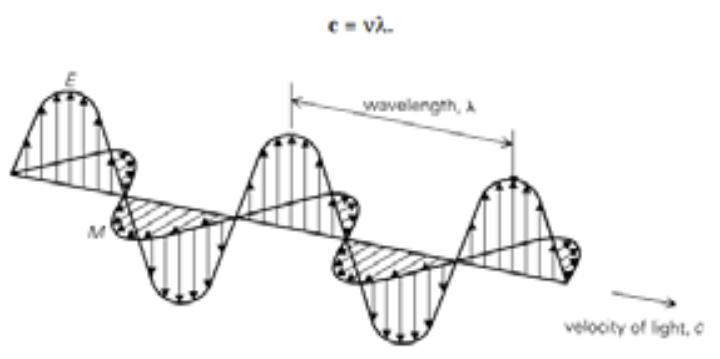

Figura 1: Ondas electromagnéticas

Fuente: (Aggarwal, Principles of remote sensing, 2004.

Existen dos teorías que describen el comportamiento de la energía electromagnética, la teoría básica de las ondas antes descrita, que indica que la energía que se propaga viaja a la velocidad de la luz con un movimiento armónico sinusoidal y la teoría de las partículas que explica que la energía electromagnética interactúa con la materia y está compuesta de partículas discretas llamadas fotones/quantos, en donde la energía del fotón es (Aggarwal, 2004):

$\mathrm{Q}=\mathrm{hc} / \lambda=\mathrm{h} \lambda$

Donde Q es la energía del fotón y h la constante de Planck.

La radiación electromagnética es referida a la propagación de la energía a través del espacio en forma de ondas (Unión Internacional de Telecomunicaciones, 2009), y el espectro electromagnético es el rango completo de todas estas radiaciones (National Aeronautics and Space Administration, 2011), que se expresan en función ya sea de la longitud de onda o de la frecuencia que es inversamente proporcional a la longitud de onda de la energía electromagnética propagada, donde a menor longitud de onda mayor frecuencia y viceversa (Canada Centre for Remote Sensing, 2016).

El límite teórico inferior es el 0 , no existiendo frecuencias negativas, la longitud de onda más pequeña posible es la longitud de Planck $\left(1 \mathrm{p} \approx 1,616199(97) * 10^{-35} \mathrm{~m}\right)$, por debajo de la cual se espera que el espacio deje de tener una geometría clásica, y el límite teórico superior es el $\infty$. (Ordóñez, 2012).

A efectos prácticos, el espectro electromagnético se extiende desde las longitudes de onda más largas incluyendo microondas y radio hasta las longitudes más cortas incluyendo Rayos Gamma y Rayos X, cubriendo así longitudes de onda de entre miles de kilómetros y la fracción del tamaño de un átomo (Canada Centre for Remote Sensing, 2016) (Ver Figura 2). 


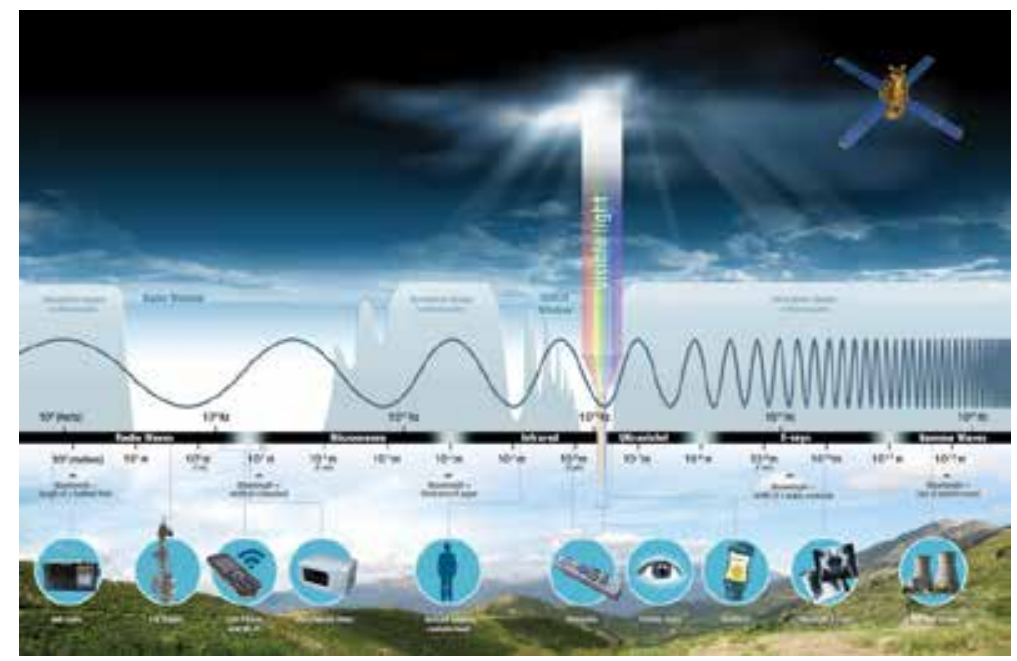

Figura 2: Espectro electromagnético

Fuente: National Aeronautic Space Agency - The Electromagnetic Spectrum, Recuperado de: https://smd-prod.s3.amazonaws.com/science-blue/s3fs-public/thumbnails/image/EMSIntroduction.jpeg

Una de las características esenciales del espectro electromagnético es que reúne las condiciones para transportar información en su proceso de propagación de las ondas, en general las señales transmitidas que utilizan frecuencias elevadas presentan distancias de propagación menores, pero gozan de una mayor capacidad de transmisión de datos, lo que implica identificar limitaciones en las aplicaciones identificadas para la cual cada banda es adecuada (Unión Internacional de Telecomunicaciones, 2009).

\section{CLASIFICACIÓN DEL ESPECTRO ELECTROMAGNÉTICO A NIVEL INTERNACIONAL Y NACIONAL}

El espectro electromagnético está dividido en diferentes regiones: Radio, Microondas, Infrarrojo, Visible, Ultravioleta, Rayos X y Rayos Gamma (National Aeronautics and Space Administration, 2014), sin embargo estas divisiones no tienen fronteras rígidas entre regiones adyacentes (Fontal, Suárez, Reyes, Bellandi, Contreras, \& Romero, 2005), por lo que existen trasposiciones entre tipos vecinos, (National Aeronautics and Space Administration, 2011) pudiendo así una misma longitud de onda quedar incluida en dos rangos diferentes (Ordóñez, 2012).

Visto que la clasificación macro del espectro electromagnético es precisa pero que los límites de sus fronteras no lo son, se pretende a través de la revisión de las diferentes propuestas generadas por diversos autores, organismos o estados que establecieron dichos rangos en función ya sea de los fines para los que destinaban su obtención o de los equipos que utilizaron para su medición, el establecer una propuesta de declaración del espectro electromagnético para Ecuador basada en dichos insumos. 


\section{CASO 1. CLASIFICACIÓN REALIZADA POR LOS ESTADOS}

Los Estados Miembros de las Naciones Unidas a través de su organismo especializado la Unión Internacional de Telecomunicaciones (UIT) ratificaron y mantienen en común el "Reglamento de Radiocomunicaciones", enmarcado en la utilización y gestión del espectro radioeléctrico a nivel nacional y respetando el marco internacional, los países miembros guían su accionar basados en el Cuadro nacional de atribución de frecuencias (se revisará en el Caso 4) que establece qué servicios radioeléctricos pueden utilizar cada una de las bandas de frecuencias y en qué condiciones (Unión Internacional de Telecomunicaciones, 2009).

Si bien es cierto que este tratado es a nivel internacional y que establece los rangos de esta sección del espectro para los Estados Miembros, no existe normativa internacional ni común entre los países para las demás secciones, existiendo casos puntuales de países que las reglamentan como España, cuyo objetivo fue establecer las disposiciones mínimas para la protección de los trabajadores, contra los riesgos para la salud y la seguridad derivados de la exposición a las radiaciones ópticas artificiales, acción ejecutada mediante el REAL DECRETO 486/2010, de 23 de abril (Instituto Nacional de Seguridad e Higiene en el Trabajo, 2010) que específica:

"Radiación ultravioleta: Radiación óptica de longitud de onda comprendida entre 100 y $400 \mathrm{~nm}$. La región ultravioleta se divide en UVA (315-400 nm), UVB (280-315 nm) y UVC (100-280 nm).

Radiación visible: La radiación óptica de longitud de onda comprendida entre $380 \mathrm{~nm}$ y $780 \mathrm{~nm}$.

Radiación infrarroja: La radiación óptica de longitud de onda comprendida entre $780 \mathrm{~nm}$ y $1 \mathrm{~mm}$. La región infrarroja se divide en IRA (780-1.400 nm), IRB (1.400-3.000 nm) e IRC (3.000 nm-1mm)."

Propiciando así el marco regulatorio para una adecuada planificación y gestión institucional y nacional en torno a riesgos de salud.

\section{CASO. 2. CLASIFICACIÓN PROPUESTA POR ALGUNOS AUTORES}

En la Tabla 1 se presenta la recopilación de algunos autores e instituciones que han establecido los rangos de cada clasificación del espectro electromagnético, basados especialmente en la utilización según su temática, incluye óptica e iluminación, salud, radio comunicaciones, sensores remotos, observación espacial y bibliografía especializada. 
Tabla 1. Clasificación del espectro electromagnético según varios autores.

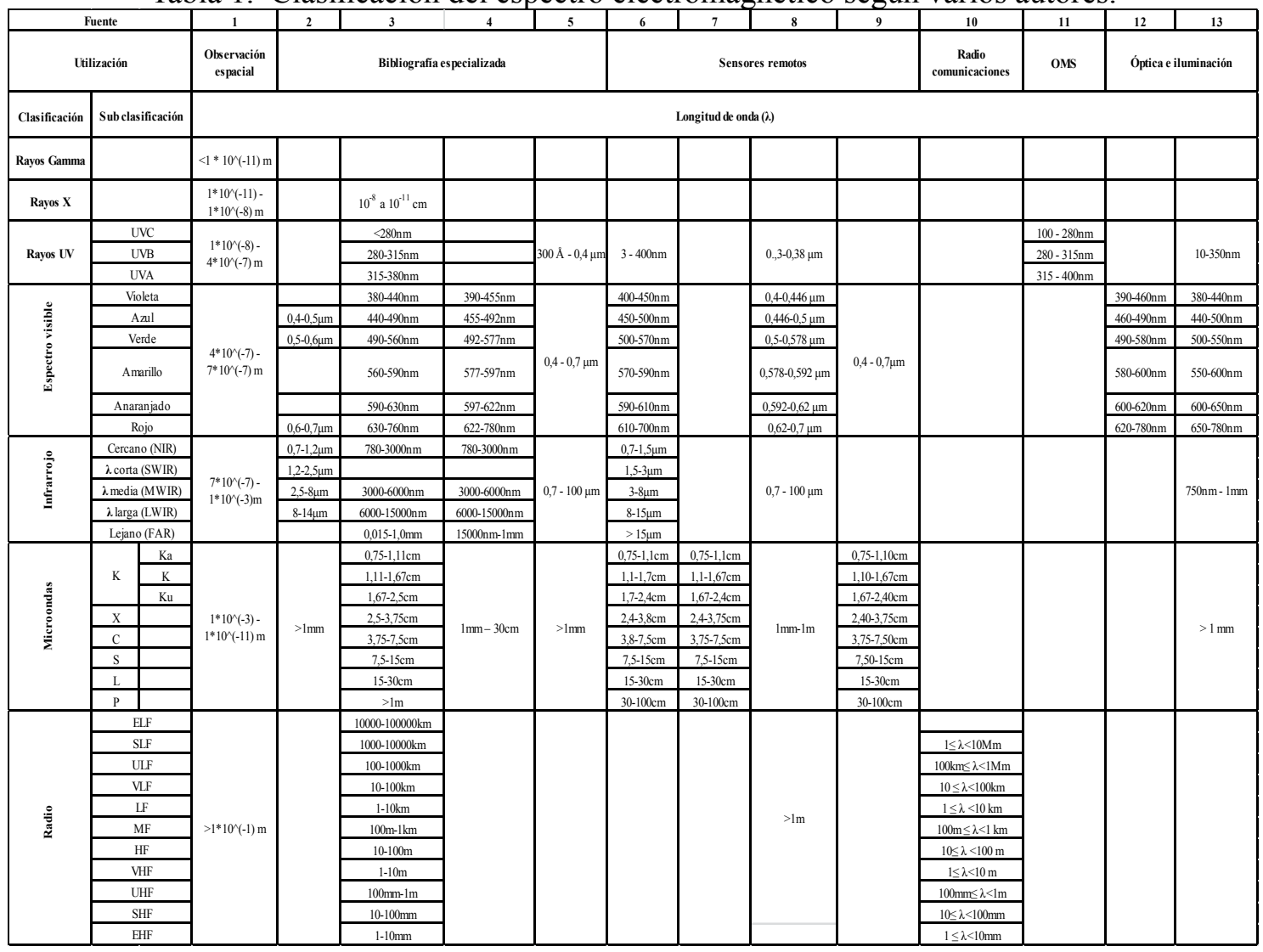

Compilado de: 1. (National Aeronautics and Space Administration, 2013), 2. (Chuvieco, 2010), 3. (Fontal, Suárez, Reyes, Bellandi, Contreras, \& Romero, 2005), 4. (Rodríguez García \& Virgós, 1999), 5. (Sobrino, 2000), 6. (Liew, 2001), 7. (Canada Centre for Remote Sensing, 2016), 8. (Aggarwal, 2004), 9. (Varón, 2012), 10. (Ordóñez, 2012) 11. (Organización Mundial de la Salud, 2002), 12. (I.E.S. Al-Ándalus de Arahal, 2016), 13. (Sirlin, 2006).

\section{CASO. 3. CLASIFICACIÓN CONFORME ORGANISMOS INTERNACIONALES.}

Existen organismos internacionales que han especificado los rangos de las regiones del espectro electromagnético básicamente en función de las posibles aplicaciones, entre ellas destacan: International Organization for Standardization - ISO (Organización Internacional de Normalización), la Commission Internationale de l'Eclairage - CIE (Comisión Internacional de Iluminación), y la International Electrotechnical Commission - IEC (Comisión Electrotécnica Internacional). La Tabla 2 presenta la clasificación realizada por la Norma ISO 21348 - Space environment (natural and artificial) Process for determining solar irradiances, cuyo propósito es crear un método estándar para determinar las irradiaciones solares para uso de sistemas espaciales (International Organization for Standarization, 2007). 
Tabla 2. Norma ISO 21348 (Space environment (natural and artificial) - Process for determining solar irradiances)

\begin{tabular}{|c|c|c|c|}
\hline $\begin{array}{l}\text { Categoría } \\
\text { espectral }\end{array}$ & $\begin{array}{c}\text { Sub categoría } \\
\text { espectral }\end{array}$ & Longitud de onda & Notas \\
\hline Rayos Gamma & & $10 \mathrm{fm} \leq \lambda<1 \mathrm{pm}$ & \\
\hline \multirow{2}{*}{ Rayos X } & & $1 \mathrm{pm} \leq \lambda 0,10 \mathrm{~nm}$ & Rayos X fuertes - Hard X rays \\
\hline & XUV & $0,10 \mathrm{~nm} \leq \lambda<10 \mathrm{~nm}$ & Rayos X débiles - Soft X rayx \\
\hline \multirow{10}{*}{ Ultravioleta } & UV & $100 \mathrm{~nm} \leq \lambda<400 \mathrm{~nm}$ & Ultravioleta \\
\hline & VUV & $10 \mathrm{~nm} \leq \lambda<200 \mathrm{~nm}$ & Ultravioleta al vacío - Vacuum Ultraviolet \\
\hline & EUV & $10 \mathrm{~nm} \leq \lambda<121 \mathrm{~nm}$ & Ultravioleta extremo - Extreme Ultraviolet \\
\hline & H Lyman- $\alpha$ & $121 \mathrm{~nm} \leq \lambda<122 \mathrm{~nm}$ & Hidrógeno Lyman - alfa \\
\hline & FUV & $122 \mathrm{~nm} \leq \lambda<200 \mathrm{~nm}$ & Ultravioleta lejano - Far Ultraviolet \\
\hline & UVC & $100 \mathrm{~nm} \leq \lambda<280 \mathrm{~nm}$ & Ultravioleta C - Ultraviolet C \\
\hline & MUV & $200 \mathrm{~nm} \leq \lambda<300 \mathrm{~nm}$ & Ultravioleta medio - Middle Ultraviolet \\
\hline & UVB & $280 \mathrm{~nm} \leq \lambda<315 \mathrm{~nm}$ & Ultravioleta B - Ultraviolet B \\
\hline & NUV & $300 \mathrm{~nm} \leq \lambda<400 \mathrm{~nm}$ & Ultravioleta cercano - Near Ultraviolet \\
\hline & UVA & $315 \mathrm{~nm} \leq \lambda<400 \mathrm{~nm}$ & Ultravioleta A - Ultraviolet A \\
\hline \multirow{7}{*}{ Visible } & \multirow{7}{*}{ VIS } & $380 \mathrm{~nm} \leq \lambda<760 \mathrm{~nm}$ & Óptico \\
\hline & & $360 \mathrm{~nm} \leq \lambda<450 \mathrm{~nm}$ & Púrpura \\
\hline & & $450 \mathrm{~nm} \leq \lambda<500 \mathrm{~nm}$ & Azul \\
\hline & & $500 \mathrm{~nm} \leq \lambda<570 \mathrm{~nm}$ & Verde \\
\hline & & $570 \mathrm{~nm} \leq \lambda<591 \mathrm{~nm}$ & Amarillo \\
\hline & & $591 \mathrm{~nm} \leq \lambda<610 \mathrm{~nm}$ & Naranja \\
\hline & & $610 \mathrm{~nm} \leq \lambda<760 \mathrm{~nm}$ & Rojo \\
\hline \multirow{5}{*}{ Infrarrojo } & IR & $760 \mathrm{~nm} \leq \lambda<1,00 \mathrm{~mm}$ & Infrarrojo \\
\hline & IR-A & $760 \mathrm{~nm} \leq \lambda<1,40 \mu \mathrm{m}$ & Infrarrojo cercano - Near Infrared \\
\hline & IR-B & $1,40 \mu \mathrm{m} \leq \lambda<3,00 \mu \mathrm{m}$ & Infrarrojo medio - Middle Infrared \\
\hline & IR-C & $3,00 \mu \mathrm{m} \leq \lambda<1,00 \mathrm{~mm}$ & Infrarrojo lejano - Far Infrared \\
\hline & & $1,00 \mathrm{~mm} \leq \lambda<15,00 \mathrm{~mm}$ & \\
\hline \multirow{9}{*}{ Microondas } & $\mathrm{W}$ & $3,00 \mathrm{~mm} \leq \lambda<5,35 \mathrm{~mm}$ & $100 \geq v>56,0 \mathrm{GHz}$ \\
\hline & $\mathrm{V}$ & $5,35 \mathrm{~mm} \leq \lambda<6,52 \mathrm{~mm}$ & $56,0 \geq v>46,0 \mathrm{GHz}$ \\
\hline & Q & $6,52 \mathrm{~mm} \leq \lambda<8,33 \mathrm{~mm}$ & $46,0 \geq v>36,0 \mathrm{GHz}$ \\
\hline & $\mathrm{K}$ & $8,33 \mathrm{~mm} \leq \lambda<27,5 \mathrm{~mm}$ & $36,00 \geq v>10,90 \mathrm{GHz}$ \\
\hline & $\mathrm{X}$ & $27,50 \mathrm{~mm} \leq \lambda<57,70 \mathrm{~mm}$ & $10,90 \geq v>5,20 \mathrm{GHz}$ \\
\hline & $\mathrm{C}$ & $48,40 \mathrm{~mm} \leq \lambda<76,90 \mathrm{~mm}$ & $6,20 \geq v>3,90 \mathrm{GHz}$ \\
\hline & $\mathrm{S}$ & $57,70 \mathrm{~mm} \leq \lambda<193,00 \mathrm{~mm}$ & $5,20 \geq v>1,55 \mathrm{GHz}$ \\
\hline & $\mathrm{L}$ & $193,00 \mathrm{~mm} \leq \lambda<769,00 \mathrm{~mm}$ & $1,550 \geq v>0,390 \mathrm{GHz}$ \\
\hline & $\mathrm{P}$ & $769,00 \mathrm{~mm} \leq \lambda<1,33 \mathrm{~m}$ & $0,390 \geq v>0,225 \mathrm{GHz}$ \\
\hline \multirow{6}{*}{ Radio } & & $0,10 \mathrm{~mm} \leq \lambda<100 \mathrm{~m}$ & Mediciones: $1000000 \leq \lambda<10000000000 \mathrm{~nm}$ \\
\hline & EHF & $1,00 \mathrm{~mm} \leq \lambda<10,00 \mathrm{~mm}$ & Frecuencia extremadamente alta $300 \geq v>30 \mathrm{GHz}$ \\
\hline & SHF & $10,00 \mathrm{~mm} \leq \lambda<100,00 \mathrm{~mm}$ & Frecuencia súper alta $30 \geq v>3 \mathrm{GHz}$ \\
\hline & UHF & $100,00 \mathrm{~mm} \leq \lambda<1,00 \mathrm{~m}$ & Frecuencia ultra alta $3000 \geq v>300 \mathrm{MHz}$ \\
\hline & VHF & $1,00 \mathrm{~m} \leq \lambda<10,00 \mathrm{~m}$ & Frecuencia muy alta $300 \geq v>30 \mathrm{MHz}$ \\
\hline & $\mathrm{HF}$ & $10,00 \mathrm{~m} \leq \lambda<100,00 \mathrm{~m}$ & Frecuencia alta $30 \geq v>3 \mathrm{MHz}$ \\
\hline
\end{tabular}


La Tabla 3 presenta la clasificación realizada por la Norma ISO 20473 - Optics and photonics - Spectral bands, cuyo objeto es aportar a la delimitación, designación y descripción de las regiones ópticas y fotónicas del espectro electromagnético (ultravioleta, visible e infrarrojo) para aplicaciones en el campo óptico y fotónico. (International Organization for Standarization, 2007).

Tabla 3. Norma ISO 20473 (Optics and photonics - Spectral bands)

\begin{tabular}{|c|c|c|c|c|c|c|}
\hline \multicolumn{2}{|c|}{ Designación de la radiación } & \multicolumn{3}{|c|}{ Designación corta } & Longitud de onda $\mathrm{nm}$ & Frecuencia THz \\
\hline \multirow{5}{*}{$\begin{array}{l}\text { Radiación } \\
\text { ultravioleta }\end{array}$} & Extreme UV & \multirow{5}{*}{ UV } & & EUV & $1-100$ & $3 * 10^{5}$ a 3000 \\
\hline & Vacuum UV & & \multirow{2}{*}{ UV-C } & VUV & $100-190$ & $3000-1580$ \\
\hline & Deep UV & & & DUV & $190-280$ & $1580-1070$ \\
\hline & Mid UV & & \multicolumn{2}{|l|}{ UV-B } & $280-315$ & $1070-950$ \\
\hline & Near UV & & \multicolumn{2}{|l|}{$U V-A^{b}$} & $315-380$ & $950-790$ \\
\hline \multicolumn{5}{|c|}{ Radiación visible, óptico } & $380-780$ & $790-385$ \\
\hline \multirow{4}{*}{ Infrarrojo } & \multirow{2}{*}{ Near IR } & \multirow{4}{*}{ IR } & IR-A & \multirow[t]{2}{*}{ NIR } & $780-1400$ & $385-215$ \\
\hline & & & IR-B & & $1400-3000$ & $215-100$ \\
\hline & Mid IR & & \multirow{2}{*}{ IR-C } & MIR & $3000-50000$ & $100-6$ \\
\hline & Far IR & & & FIR & $50000-1000000$ & $6-0.3$ \\
\hline
\end{tabular}

La Tabla 4 muestra los márgenes establecidos por la CIE, organización líder en desarrollo de estándares y procedimientos básicos de metrología en los campos de luz e iluminación (International Commission on Illumination, 2017), y la IEC, organización líder mundial en la preparación y publicación de estándares internacionales para todas las tecnologías eléctricas y electrónicas relacionadas. (International Electrotechnical Commission, 2016).

Protagonizando

Tabla 4. Clasificaciones del espectro electromagnético realizadas por la IEC y la CIE

\begin{tabular}{|c|c|c|c|}
\hline \multirow{2}{*}{$\begin{array}{c}\text { Categoría } \\
\text { espectral }\end{array}$} & $\begin{array}{c}\text { Sub categoría } \\
\text { espectral }\end{array}$ & \multicolumn{2}{c|}{ Rango de longitud de onda } \\
\cline { 1 - 1 } & UVC & $100-280 \mathrm{~nm}$ & CIE \\
Ultravioleta & UVB & $280-315 \mathrm{~nm}$ & $100-280 \mathrm{~nm}$ \\
& UVA & $315-400 \mathrm{~nm}$ & $280-315 \mathrm{~nm}$ \\
Visible & $V I S$ & $360-400$ a $760-830 \mathrm{~nm}$ & $315-400 \mathrm{~nm}$ \\
& IR-A & $780-1400 \mathrm{~nm}$ & $400-700 \mathrm{~nm}$ \\
Infrarrojo & IR-B & $1,4-3 \mu \mathrm{m}$ & $700 \mathrm{~nm}-3 \mu \mathrm{m}$ \\
& IR-C & $3 \mu \mathrm{m} \mathrm{a} 1 \mathrm{~mm}$ & $1,4-3 \mu \mathrm{m}$ \\
& & & $3 \mu \mathrm{m} \mathrm{a} 1 \mathrm{~mm}$ \\
\hline
\end{tabular}

La Tabla 5 muestra los rangos del espectro radioeléctrico, convencionalmente establecido desde $3 \mathrm{kHz}$ hasta $3000 \mathrm{GHz}$, (Unión Internacional de Telecomunicaciones, 1999), a través de la UIT, organismo especializado de las Naciones Unidas para las Tecnologías de la información y comunicación que se encarga de la atribución del espectro radioeléctrico y las órbitas de satélite a escala mundial, elaborando normas técnicas para garantizar la interconexión 
continua de las redes y las tecnologías (Unión Internacional de Telecomunicaciones, 2013).

Tabla 5. Bandas y sub-bandas de frecuencia conforme la UIT

\begin{tabular}{|ccccc|}
\hline $\begin{array}{c}\text { Número } \\
\text { de la } \\
\text { banda } \\
4\end{array}$ & Símbolos & $\begin{array}{c}\text { Gama de frecuencias (ex- } \\
\text { cluido el límite inferior pero } \\
\text { incluido el superior) } \\
3-30 \mathrm{kHz}\end{array}$ & $\begin{array}{c}\text { Subdivisión métrica } \\
\text { correspondiente } \\
\text { Ondas miriamétricas }\end{array}$ & $\begin{array}{c}\text { Abreviaturas } \\
\text { métricas para } \\
\text { las bandas } \\
\text { B.Mam }\end{array}$ \\
\hline 5 & LF & $30-300 \mathrm{kHz}$ & Ondas kilométricas & B.km \\
\hline 6 & MF & $300-3000 \mathrm{kHz}$ & Ondas hectométricas & B.hm \\
\hline 7 & HF & $3-30 \mathrm{MHz}$ & Ondas decamétricas & B.dam \\
\hline 8 & VHF & $30-300 \mathrm{MHz}$ & Ondas métricas & B.m \\
\hline 9 & UHF & $300-3000 \mathrm{MHz}$ & Ondas decimétricas & B.dm \\
\hline 10 & SHF & $3-30 \mathrm{GHz}$ & Ondas centimétricas & B.cm \\
\hline 11 & EHF & $30-300 \mathrm{GHz}$ & Ondas milimétricas & B.mm \\
\hline 12 & & $300-3000 \mathrm{GHz}$ & Ondas decimilimétricas & B.dmm \\
\hline
\end{tabular}

Finalmente para complementar la Tabla 5, se presenta la Tabla 6 que muestra algunos de los usos relacionados a las diversas bandas de frecuencias radioeléctricas en función a las características de propagación. (Unión Internacional de Telecomunicaciones, 2009), aportando así a todos los procesos relacionados con actividades de gestión de este recurso.

Tabla 6. Características de propagación de las bandas de frecuencia radioeléctricas

\begin{tabular}{|c|c|c|c|c|c|}
\hline Banda & $\begin{array}{l}\text { Rango de } \\
\text { frecuencias }\end{array}$ & Alcance & Utilización & $\begin{array}{l}\text { Anchura de } \\
\text { banda }\end{array}$ & Interferencia \\
\hline $\begin{array}{l}\text { Ondas miri- } \\
\text { amétricas }\end{array}$ & 3 a $30 \mathrm{kHz}$ & Miles de km & $\begin{array}{l}\text { Radionavegación de } \\
\text { largo alcance }\end{array}$ & Muy estrecha & $\begin{array}{l}\text { De amplia } \\
\text { distribución }\end{array}$ \\
\hline $\begin{array}{l}\text { Ondas ki- } \\
\text { lométricas }\end{array}$ & 30 a $300 \mathrm{kHz}$ & Miles de km & $\begin{array}{l}\text { Como las comunica- } \\
\text { ciones estratégicas en } \\
\text { ondas miriamétricas }\end{array}$ & Muy estrecha & $\begin{array}{l}\text { De amplia } \\
\text { distribución }\end{array}$ \\
\hline $\begin{array}{l}\text { Ondas hec- } \\
\text { tométricas }\end{array}$ & $\begin{array}{c}300 \text { a } 3000 \\
\mathrm{kHz}\end{array}$ & $2-3000 \mathrm{~km}$ & $\begin{array}{l}\text { Como las comunica- } \\
\text { ciones estratégicas en } \\
\text { ondas miriamétricas }\end{array}$ & Moderada & $\begin{array}{l}\text { De amplia } \\
\text { distribución }\end{array}$ \\
\hline $\begin{array}{l}\text { Ondas de- } \\
\text { camétricas }\end{array}$ & 3 a $30 \mathrm{MHz}$ & $\begin{array}{l}\text { Hasta } 1000 \\
\mathrm{~km}\end{array}$ & $\begin{array}{l}\text { Radiodifusión y punto a } \\
\text { punto a nivel mundial }\end{array}$ & Amplia & $\begin{array}{l}\text { De amplia } \\
\text { distribución }\end{array}$ \\
\hline $\begin{array}{l}\text { Ondas } \\
\text { métricas }\end{array}$ & $\begin{array}{c}30 \text { a } 300 \\
\mathrm{MHz}\end{array}$ & $2-300 \mathrm{~km}$ & $\begin{array}{l}\text { Radiodifusión, PCS, } \\
\text { Móvil, WAN }\end{array}$ & Muy amplia & Confinada \\
\hline $\begin{array}{l}\text { Ondas dec- } \\
\text { imétricas }\end{array}$ & $\begin{array}{l}300 \text { a } 3000 \\
\quad \mathrm{MHz}\end{array}$ & $<100 \mathrm{~km}$ & $\begin{array}{l}\text { Radiodifusión, PCS, } \\
\text { Móvil, WAN }\end{array}$ & Muy amplia & Confinada \\
\hline $\begin{array}{l}\text { Ondas cen- } \\
\text { timétricas }\end{array}$ & 3 a $30 \mathrm{GHz}$ & $\begin{array}{l}\text { Varia de } \\
30 \mathrm{~km} \mathrm{a} \\
2000 \mathrm{~km}\end{array}$ & $\begin{array}{l}\text { Radiodifusión, PCS, } \\
\text { Móvil, WAN, comunica- } \\
\text { ciones por satélite }\end{array}$ & $\begin{array}{l}\text { Muy amplia } \\
\text { hasta } 1 \mathrm{GHz}\end{array}$ & Confinada \\
\hline $\begin{array}{c}\text { Ondas } \\
\text { milimétricas }\end{array}$ & 30 a $300 \mathrm{GHz}$ & $\begin{array}{l}\text { Varia de } \\
20 \mathrm{~km} \mathrm{a} \\
2000 \mathrm{~km}\end{array}$ & $\begin{array}{l}\text { Radiodifusión, punto a } \\
\text { punto, PCS y comunica- } \\
\text { ciones por satélite }\end{array}$ & $\begin{array}{l}\text { Muy amplia } \\
\text { hasta } 10 \mathrm{GHz}\end{array}$ & Confinada \\
\hline
\end{tabular}




\section{CASO. 4. CLASIFICACIÓN Y USO DEL ESPECTRO ELECTROMAGNÉTICO EN ECUADOR Y EN LOS INSTITUTOS DE LA DEFENSA.}

A nivel Ecuador no existe una declaración de los rangos del espectro electromagnético, pero si una regulación en cuanto a la asignación y uso de frecuencias del espectro radioeléctrico acogiendo los criterios de la Unión Internacional de Telecomunicaciones como Estado Miembro de las Naciones Unidas.

En el proceso de gestión y regulación del uso del espectro, Ecuador ha regulado su utilización mediante el Registro Oficial Nro. 439 del 18 de febrero de 2015 en el que se promulga la Ley Orgánica de Telecomunicaciones, cuyo objeto es "desarrollar, el régimen general de telecomunicaciones y del espectro radioeléctrico como sectores estratégicos del Estado que comprende las potestades de administración, regulación, control y gestión en todo el territorio nacional, bajo los principios y derechos constitucionalmente establecidos" (Registro Oficial del Ecuador, 2015), se suma a esta gestión el Plan Nacional de Frecuencias de Ecuador 2012, que establece la atribución de las bandas de frecuencias a los diferentes servicios de radiocomunicaciones (Consejo Nacional de Telecomunicaciones, 2012), pero como se observa sólo se atiende esta sección del espectro en cuanto procesos de regulación.

Si bien no existe una normativa o regulaciones respecto de las otras secciones del espectro, si existen varias instituciones del Estado que han enfocado sus esfuerzos en obtener información y generar productos y aplicaciones en función de este recurso, entre ellas nos referiremos a las Instituciones de la Defensa del Ecuador, Tabla 7:

Instituto Geográfico Militar - IGM: Responsable de gestionar y ejecutar las actividades de investigación aplicada, generación y control de geoinformación y transferencia de conocimiento y tecnología en los ámbitos de geodesia, geomática, geografía, cartografía y seguridad documentaria (Instituto Geográfico Militar, 2016).

Instituto Oceanográfico de la Armada - INOCAR: Planificar, dirigir, coordinar y controlar las actividades técnicas y administrativas relacionadas con el Servicio de Hidrografía, Navegación, Oceanografía, Meteorología, Ciencias del Mar, Señalización Náutica, así como la administración del material especializado con su actividad (Instituto Oceanográfico de la Armada, 2017).

Instituto Espacial Ecuatoriano - IEE: Mantener e impulsar la investigación científica y desarrollo tecnológico espacial y el incremento de la cultura aeroespacial, que contribuyan a la Defensa y Desarrollo Nacional (Instituto Espacial Ecuatoriano, 2017).

Instituto Antártico del Ecuador - INAE: Fomentar y mantener la proyección geopolítica del país y la participación permanente en las actividades de investigación científica, en el contexto del Sistema del Tratado Antártico (Instituto Antártico Ecuatoriano, 2017). 
Tabla 7. Regiones del espectro electromagnéticas capturadas y gestionadas por las Instituciones de Defensa y el CONATEL.

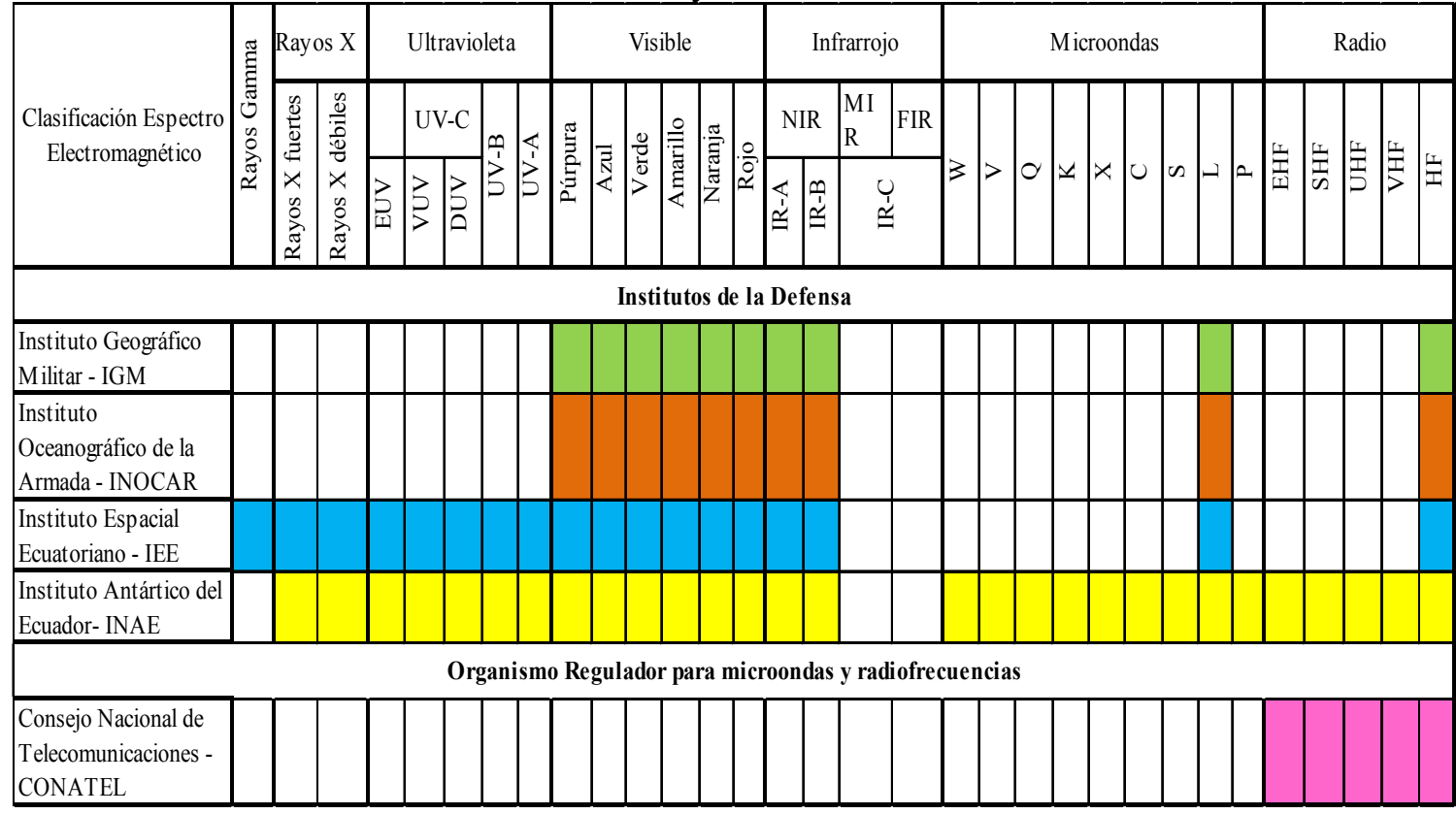

\section{ANÁLISIS}

De acuerdo a la información presentada en las Tablas 1, 2, 3 y 4 se verifica que los rangos establecidos para cada sub clasificación del espectro electromagnético son más o menos arbitrarios, y su asignación no corresponde estrictamente con la temática en la que se usa, ya que existen diferentes rangos establecidos dentro de las mismas aplicaciones. En gran medida esto se explicaría debido a la capacidad de producir o detectar cada región del espectro, así como la capacidad tecnológica de producir fuentes de radiación apropiadas y detectores o medidores sensibles a las respectivas regiones (Fontal, Suárez, Reyes, Bellandi, Contreras, \& Romero, 2005).

Respecto a los posibles límites citados en las Tablas 1, 2, 3 y 4 para cada sub clasificación se puede indicar que: 1) existe una clara coherencia en la asignación de los límites de cada sub clasificación ya que si bien los límites no son exactamente iguales todos sus valores están en los mismos rangos, 2) a nivel de superposiciones se identifican dos instancias, un primer grupo entre rayos UV, Visible e Infrarrojo y un segundo grupo entre Microondas y Radio, pero claramente diferenciados entre si y de los Rayos X y Gamma y, 3) en la generalidad de las clasificaciones la determinación de los rangos para los límites del Ultravioleta son coincidentes en sus tres principales clasificaciones, rayos UVC, UVB y UVA, siendo el UVA específicamente el que presenta el traslape con el Visible en el púrpura, lo que indicaría que su identificación y delimitación es mucho más clara a pesar de las posibles aplicaciones o equipos utilizados. 
En lo referente a los procesos de gestión a nivel nacional, y conforme se observa en la Tabla 7, si bien se ha podido identificar las secciones en las que trabajan dichas instituciones no se ha podido especificar exactamente el ancho de banda captado en cada sección o si abarcan la totalidad del rango, lo que finalmente puede conllevar a no poder comparar con exactitud productos, resultados e inclusive equipos. Esto finalmente ratifica la necesidad de establecer mecanismos normativos que permitan efectivizar los esfuerzos realizados en cuanto a la gestión del espectro a fin de evitar la sobreposición de competencias y desarrollos aislados de objetivos y productos, que produzcan desfases técnicos, institucionales y eviten un avance progresivo de una correcta planeación (Estrella, 2015), ya sea por procesos no vinculados o interrelacionados, o a su vez por la ausencia de mecanismos que como en este caso permitan delimitar con precisión los rangos de acción de cada institución respecto del espectro y enfocar esfuerzos en secciones no gestionadas. Adicionalmente se observa que la mayor concentración de captura de datos y gestión se ha desarrollado por igual en torno al espectro visible, infrarrojo cercano, microondas en la banda $\mathrm{L}$ y radio en la banda $\mathrm{HF}$, es decir en las ventanas atmosféricas (Ver Figura 4), que son idóneas para realizar procesos de teledetección (Chuvieco, 2010), y de igual manera en lo relacionado a la recepción de señal GPS en microondas y radio, dejando por fuera las otras clasificaciones, lo que conlleva el disminuir el ámbito de investigación y generación de aplicaciones y productos.

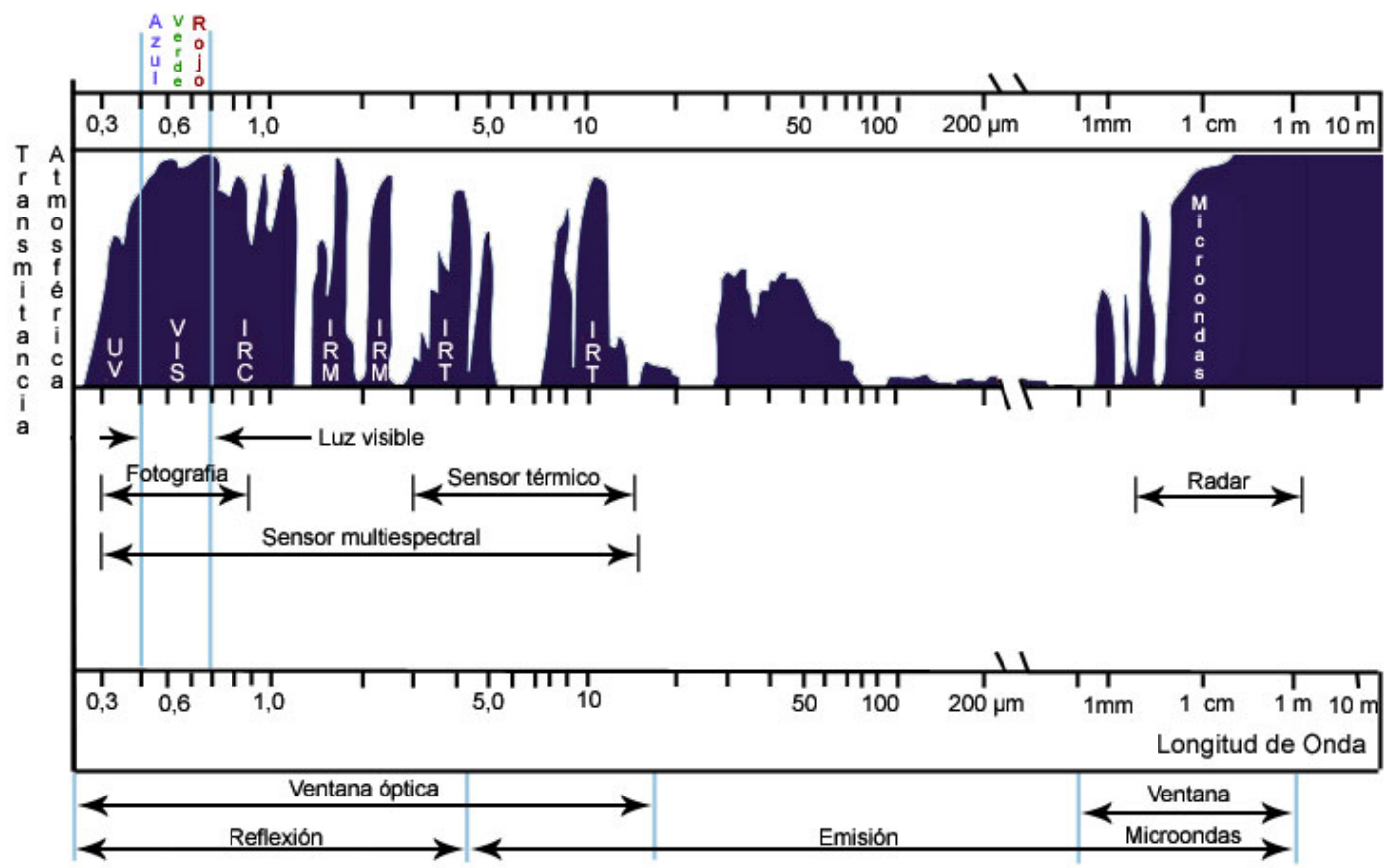

Figura 4. Interacción de la radiación electromagnética con la atmósfera - ventanas atmosféricas Fuente: http://concurso.cnice.mec.es/cnice2006/material121/unidad1/atmos_td.htm

Considerando que las cuatro instituciones analizadas enfocan la mayor parte de sus esfuerzos en los ámbitos de teledetección y que no existe una reglamentación del espectro 
en dichas sub clasificaciones se plantea un ejemplo de los posibles resultados obtenidos al manejar diferentes rangos para las mismas aplicaciones, en el supuesto por ejemplo de buscar medir el Índice de vegetación diferencial normalizado utilizando diferentes imágenes satelitales del mismo lugar:

Tabla 8. Cálculo del Índice de vegetación diferencial normalizado.

\begin{tabular}{|c|c|c|c|c|}
\hline & $\begin{array}{c}\text { Landsat } \\
\text { Rango } \mu \mathrm{m}\end{array}$ & $\begin{array}{c}\text { Spot } \\
\text { Rango } \mu \mathrm{m}\end{array}$ & $\begin{array}{c}\text { Aster } \\
\text { Rango } \mu \mathrm{m}\end{array}$ & $\begin{array}{c}\text { Respuesta } \\
\text { espectral } \mu \mathrm{m}\end{array}$ \\
\hline Rojo & $0,630-0,680$ & $0,61-0,68$ & $0,63-0,69$ & 0,62 \\
\hline Infrarrojo Cercano & $0,845-0,885$ & $0,78-0,89$ & $0,76-0,86$ & 0,85 \\
\hline NDVI $=(($ IRC-R $)) /(I R C+R)$ & NO APLICARÍA & 0,156 & NO APLICARÍA & \\
\hline
\end{tabular}

Nota: sólo las celdas en color verde abarcarían los valores medidos

Si bien este es un ejemplo básico, brinda una clara idea de los posibles resultados o escenarios que se pudiesen presentar no sólo en este caso sino en todos aquellos donde se comparen resultados, productos e inclusive equipos no sólo en el ámbito de las ciencias geoespaciales, sino de todas aquellas que hagan uso del espectro electromagnético sin tener un punto de partida base sobre el cual hacer referencia, permitiendo evidenciar la necesidad de siempre contar con un instrumento normativo que sirva de guía y referencia para posibles productos y estudios.

\section{PROPUESTA DE CLASIFICACIÓN DEL ESPECTRO ELECTROMAGNÉTICO PARA ECUADOR}

Una vez revisada la información descrita y evidenciado la clara necesidad de establecer los rangos del espectro electromagnético a fin de evitar duplicación de esfuerzos y recursos humanos, técnicos y económicos a la hora de adquirir y gestionar información del espectro, se ha generado una propuesta de clasificación del espectro electromagnético para Ecuador, que permita universalizar los criterios, lenguaje, información y comparar resultados con precisión al momento de trabajar con dicho recurso.

Para tales fines se ha consolidado lo expuesto en las Normas ISO, 21348, 20473 y se ha acogido las recomendaciones de la Asamblea de Radiocomunicaciones de la Unión Internacional de Telecomunicaciones adoptada y adaptada por el Ecuador mediante la Ley Orgánica de Telecomunicaciones y el Plan Nacional de Telecomunicaciones, conforme se muestra en la Tabla 9, priorizando la utilización de los rangos en función del objetivo para el cual fue creada cada norma o recomendación. 
Tabla 9. Propuesta de declaración del espectro electromagnético para Ecuador

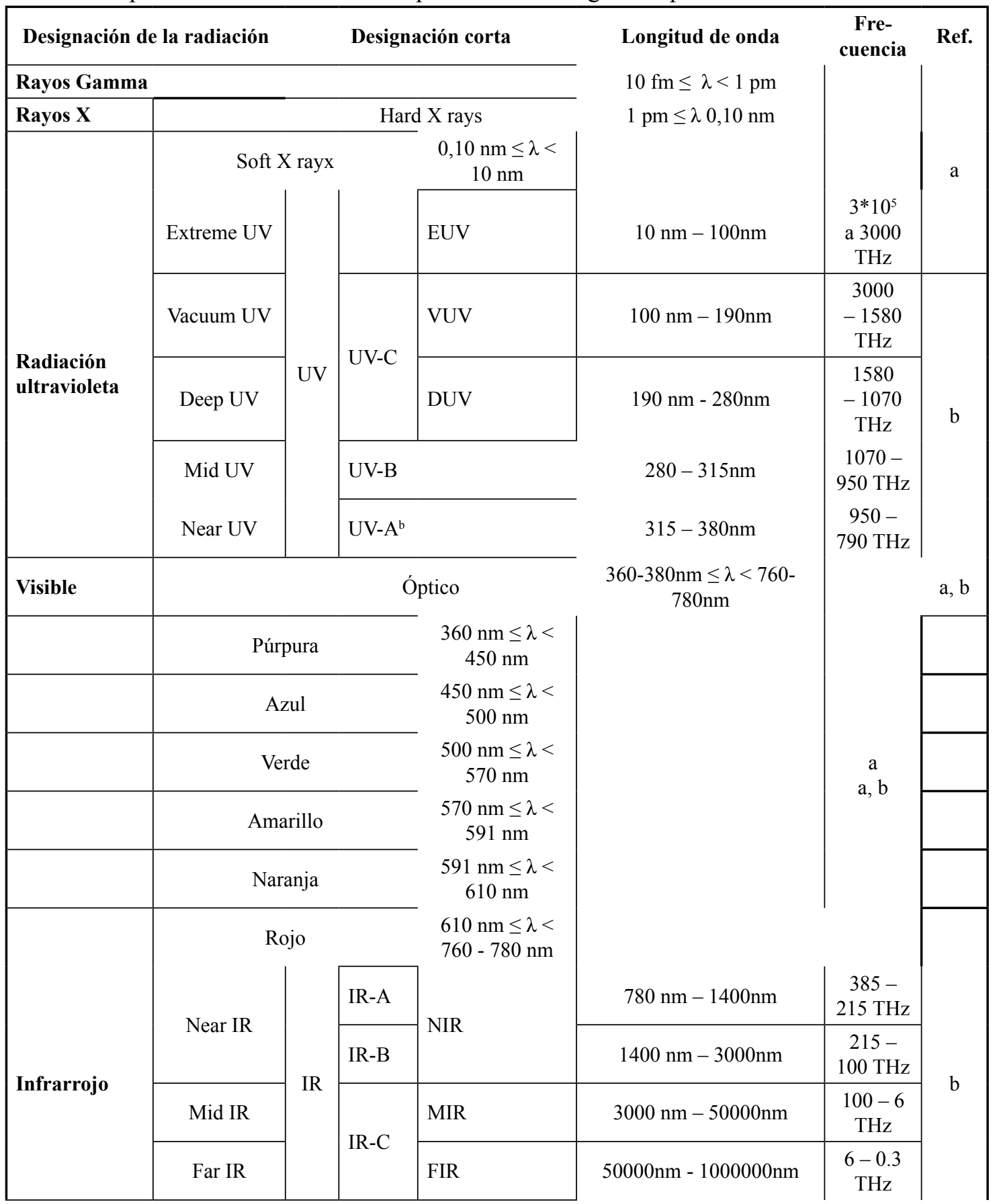




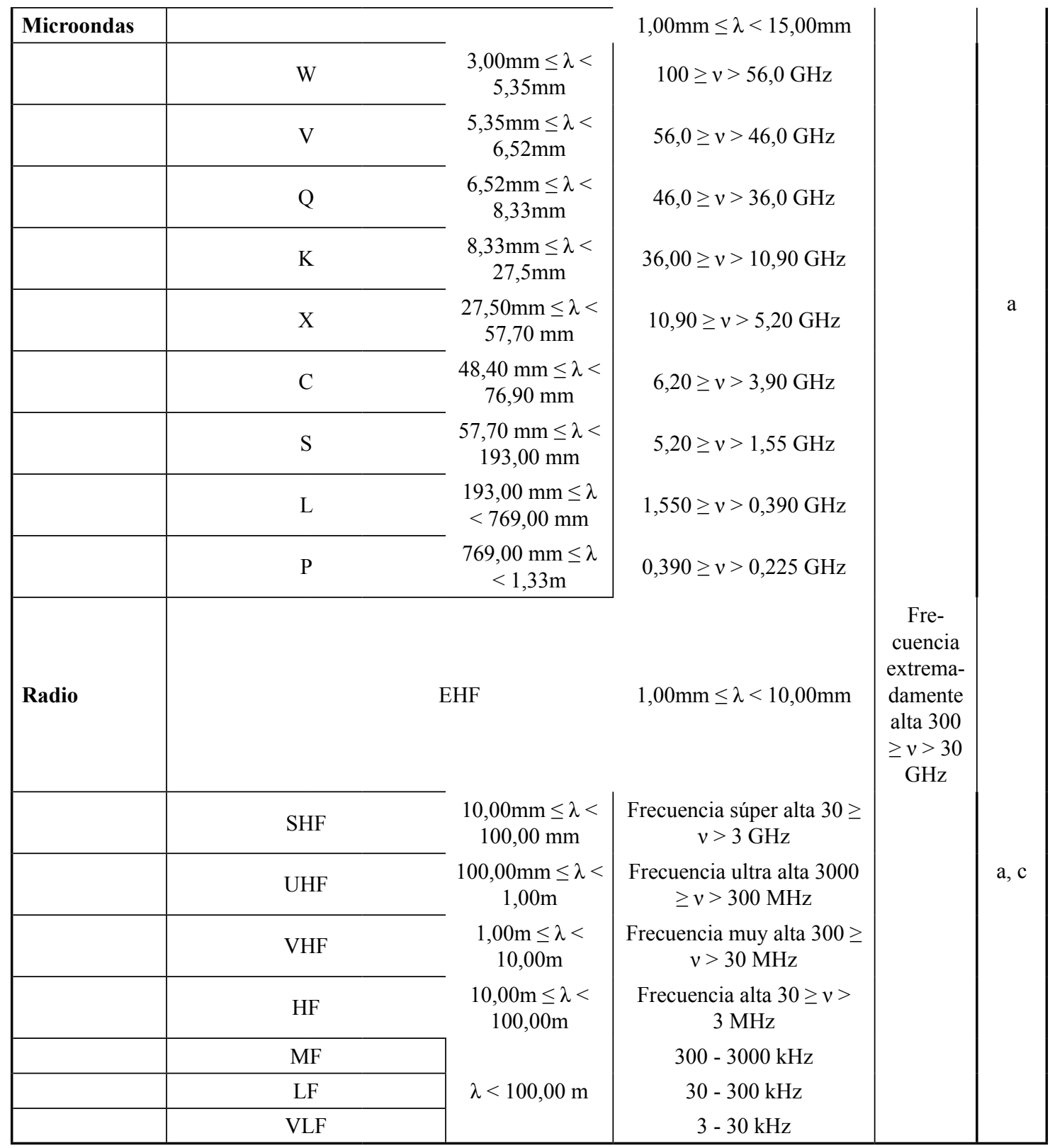

La estructuración de la Tabla 9 está generada de la siguiente manera: Norma ISO 20473 (a) para Ultravioleta, Infrarrojo y los rangos internos del Visible, Norma ISO 21348 (b) para Rayos X, Gamma, Microondas, Norma ISO 21348 y 20473 para los límites de inicio y fin del Visible y, Norma ISO 21348 y Plan Nacional de Telecomunicaciones (c) para Radio.

En base a esta propuesta se plantea estandarizar principalmente el lenguaje técnico utilizado a la hora de generar productos, adquirir equipos o centrar esfuerzos y recursos técnicos y humanos que hagan uso del espectro, de manera tal que se efectivicen los esfuerzos 
realizados en cuanto a la gestión y uso de este recurso y se incentive la distribución y correcta asignación de funciones a fin de evitar la sobreposición de competencias y desarrollos aislados en las instituciones del estado, así también como que se genere una línea base que permita a los usuarios en general, manejar información basada en estándares internacionales e incorpore un valor agregado a los posibles productos o desarrollos que se presenten.

Se destaca también la necesidad a nivel país y especialmente a nivel de Defensa y de Seguridad Nacional de contar con una caracterización del espacio electromagnético como la presente propuesta, que permita a los involucrados y tomadores de decisiones gestionar y proponer soluciones que ayuden a disminuir los riesgos de un ataque electrónico (EW - Electronic Warfare o Guerra Electrónica), que engloba cualquier acción militar que involucre el espectro electromagnético para controlar su uso, bajo la consideración de que el ciberespacio (es un ámbito caracterizado por el uso de la electrónica y el espectro electromagnético para almacenar, modificar e intercambiar datos a través de los sistemas en red y la infraestructura física asociada), es llamado el quinto dominio de la guerra (Clarke \& Knake, 2011), y la ciberseguridad afecta al bienestar digital de la sociedad, de las organizaciones y de los países y, en particular, afecta a distintas dimensiones: política, social, económica, legal, justicia y policial, técnica y de gestión (Unión Internacional de Telecomunicaciones, 2008).

La seguridad del ciberespacio es un objetivo estratégico de la seguridad nacional (Unión Internacional de Telecomunicaciones, 2008).

\section{REFERENCIAS:}

Aggarwal, S. (2004). Principles of remote sensing. Satellite remote sensing and GIS applications in agricultural meteorology (pág. 23). Switzerland: World Meteorological Organisation.

Canada Centre for Remote Sensing. (17 de Agosto de 2016). Fundamentals of Remote Sensing. Recuperado el 27 de Octubre de 2016, de http://www.nrcan.gc.ca/earth-sciences/geomatics/ satellite-imagery-air-photos/satellite-imagery-products/educational-resources/9309

Chuvieco, E. (2010). Teledetección ambiental: La observación de la tierra desde el espacio. Barcelona: Ariel.

Clarke, R. A., \& Knake, R. K. (2011). Guerra en la red. Los nuevos campos de batalla. Barcelona: Ariel.

Consejo Nacional de Telecomunicaciones. (2012). Plan Nacional de Frecuencias. Recuperado el 29 de Noviembre de 2016, de http://www.arcotel.gob.ec/wp-content/uploads/ downloads/2013/07/plan_nacional_frecuencias_2012.pdf

Estrella, C. (17 de Junio de 2015). PROPUESTA DE FORMULACIÓN PARA LAESTRUCTURA ESPACIAL DEL ECUADOR, Y LA APLICACIÓN DE LA MISMA, A LA EVALUACIÓN DE LA VULNERABILIDAD EN LA INFRAESTRUCTURA DE COMUNICACIÓN VIAL, USANDO TECNOLOGÍA GEOESPACIAL. Córdoba, Argentina.

Fontal, B., Suárez, T., Reyes, M., Bellandi, F., Contreras, R., \& Romero, I. (2005). El Espectro Electromagnético y sus Aplicaciones. Mérida: Escuela Venezolana para la Enseñanza de la Química. 
I.E.S. Al-Ándalus de Arahal. (Septiembre de 2016). La luz y las ondas electromagnéticas. Recuperado el 28 de Noviembre de 2016, de http://www.iesalandalus.com/joomla3/images/ stories/FisicayQuimica/Fis2B/t6_optica.pdf

Instituto Antártico Ecuatoriano. (21 de Septiembre de 2017). Instituto Antártico Ecuatoriano Misión - Visión - Objetivos. Recuperado el 08 de Diciembre de 2016, de http://www.inae. gob.ec/index.php/mision/

Instituto Espacial Ecuatoriano. (21 de Septiembre de 2017). Instituto Espacial Ecuatoriano - Valores/Misión/Visión. Recuperado el 08 de Diciembre de 2016, de http://www. institutoespacial.gob.ec/valores/

Instituto Geográfico Militar. (14 de Noviembre de 2016). NOTIGM. Recuperado el 16 de Noviembre de 2016, de Instituto Geográfico Militar: http:/www.noti.igm.gov.ec/notigm/ index.php?option=com_content\&view $=$ category\&layout=blog\&id=10\&Itemid $=19$

Instituto Nacional de Seguridad e Higiene en el Trabajo. (24 de Abril de 2010). Legislación consolidada. Recuperado el 30 de Noviembre de 2016, de http://www.boe.es/buscar/act. php?id=BOE-A-2010-6485

Instituto Oceanográfico de la Armada. (21 de Septiembre de 2017). Instituto Oceanográfico de la Armada - Misión y funciones. Recuperado el 08 de Diciembre de 2016, de http://www. inocar.mil.ec/web/index.php/institucion/mision-y-funciones

International Commission on Illumination. (12 de Septiembre de 2017). CIE - International Commission on Illumination. Recuperado el 12 de Septiembre de 2017, de http://www.cie. co.at/index.php/LEFTMENUE/About+us/General+Information

International Electrotechnical Commission. (12 de Noviembre de 2016). IEC - About the IEC. Recuperado el 12 de Noviembre de 2016, de http://www.iec.ch/about/?ref=menu

International Organization for Standarization. (15 de Abril de 2007). ISO 20473:2007(en) Optics and photonics - Spectral bands. Recuperado el 28 de Octubre de 2016, de https://www.iso. org/obp/ui/\#iso:std:iso:20473:ed-1:v1:en

International Organization for Standarization. (01 de Mayo de 2007). ISO 21348:2007(en) Space envrionment (natural and artificial) - Process for determining solar irradiances. Recuperado el 28 de Octubre de 2016, de https://www.iso.org/obp/ui/\#iso:std:iso:21348:ed-1:v1:en

Liew, S. (2001). Electromagnetic Radiation - Centre for Remote Imaging, Sensing and Processing. Recuperado el 15 de Noviembre de 2016, de http://www.crisp.nus.edu.sg/ research/tutorial/ em.htm

National Aeronautics and Space Administration. (11 de Marzo de 2011). Earth Observatory. Recuperado el 11 de Octubre de 2016, de http://earthobservatory.nasa.gov/Features/ RemoteSensing/remote_03.php

National Aeronautics and Space Administration. (05 de Noviembre de 2013). Regions of the Electromagnetic Spectrum. Recuperado el 7 de Noviembre de 2016, de https://imagine.gsfc. nasa.gov/science/toolbox/spectrum_chart.html

National Aeronautics and Space Administration. (14 de Noviembre de 2014). The Electromagnetic Spectrum. Recuperado el 18 de Octubre de 2016, de https://imagine.gsfc.nasa.gov/science/ toolbox/emspectrum1.html

Ordóñez, J. L. (2012). Espectro electromagnético y espectro radioeléctrico. Manual formativo de ACTA, 17-31.

Organización Mundial de la Salud. (2002). Indice UV solar mundial - World Health Organization. Recuperado el 9 de Noviembre de 2016, de http://www.who.int/uv/publications/en/uvispa. pdf 
Registro Oficial del Ecuador. (18 de Febrero de 2015). Tercer Suplemento -- Registro Oficial No 439 -- Miércoles 18 de febrero de 2015 -- 3. Quito, Pichincha, Ecuador.

Rodríguez García, J., \& Virgós, J. (1999). Fundamentos de óptica ondulatoria. Oviedo: Servicio de Publicaciones de la Universidad de Oviedo.

Sirlin, E. (2006). La Luz en el teatro, manual de iluminación. Buenos Aires: Atuel.

Sobrino, J. A.-G. (2000). Teledetección. Valencia: Servicio de Publicaciones, Universidad de Valencia.

Unión Internacional de Telecomunicaciones. (01 de Enero de 1999). RECOMENDACIÓN UIT-R V.431-6. Recuperado el 08 de Diciembre de 2016, de NOMENCLATURA DE LAS BANDAS DE FRECUENCIAS Y DE LAS LONGITUDES DE ONDA EMPLEADAS EN TELECOMUNICACIONES: https://www.itu.int/dms_pubrec/itu-r/rec/v/R-REC-V.431-6199304-S!!PDF-S.pdf

Unión Internacional de Telecomunicaciones. (Abril de 2008). SERIE X: REDES DE DATOS, COMUNICACIONES DE SISTEMAS ABIERTOS Y SEGURIDAD, Seguridad en el ciberespacio - Ciberseguridad, Aspectos generales de la ciberseguridad. Recomendación UIT-T X.1205. Recuperado el 21 de Marzo de 2016, de https://www.itu.int/rec/T-RECX.1205-200804-I/es

Unión Internacional de Telecomunicaciones. (26 de Junio de 2009). Módulo 5. Gestión del espectro radioeléctrico. Recuperado el 14 de Noviembre de 2016, de www.ictregulationtoolkit.org/ action/document/download?document_id=3782

Unión Internacional de Telecomunicaciones. (Julio de 2013). Sobre la UIT. Recuperado el 12 de Noviembre de 2016, de http://www.itu.int/es/about/Pages/default.aspx

Varón, F. G. (2012). Modelamiento estructural de la zona límite entre la microplaca de Panamá y el bloque norandino a partir de la interpretación de imágenes de radar, cartografía geológica, anomalías de campos potenciales y líneas sísmicas. Capítulo 3. Interpretación de imág. Recuperado el 10 de Noviembre de 2016, de http://www.bdigital.unal.edu. co/8848/2/194358.2012.Parte_2.pdf 